\title{
Technological process for production of persimmon and strawberry vinegars
}

This article was published in the following Dove Press journal:

International Journal of Wine Research

20 August 2010

Number of times this article has been viewed

\section{Claudio Hidalgo' \\ Estibaliz Mateo' \\ Ana Belen Cerezo ${ }^{2}$ \\ Maria-Jesús Torija' \\ Albert Mas'}

'Biotecnologia Enològica, Departament de Bioquimica i Biotecnologia, Facultat d'Enologia, Universitat Rovira i Virgili, Marcel.li Domingo, Tarragona; ${ }^{2}$ Área de Nutrición y Bromatología, Facultad de Farmacia, Universidad de Sevilla, Sevilla, Spain
Correspondence: Albert Mas

Biotecnologia Enològica,

Departament de Bioquimica i

Biotecnologia, Facultat d'Enologia,

Universitat Rovira i Virgili, Marcel.li

Domingo s/n, 43007, Tarragona, Spain

Tel +34977558688

$\mathrm{Fax}+34977558232$

Email albert.mas@urv.cat

\begin{abstract}
Fruit surplus is common in intensive agriculture in many countries. This ecologic and economic problem requires alternative uses to be found for fruit. The aim of this study was to use surplus fruit to produce vinegar by traditional methods (alcoholic fermentation and acetification) from persimmon and strawberry. The process was performed with naturally occurring microorganisms and compared with inoculated commercial wine yeast for alcoholic fermentation. Alcoholic fermentation proceeded faster when inoculated due to the length of the lag phases observed in spontaneous fermentations. The alcoholic fermentations of strawberry mash were faster than those of persimmon mash. In contrast, acetifications were much faster in persimmon (30 days) than in strawberry (70 days), in the latter some acetifications were not finished. From the technologic point of view, to produce persimmon and strawberry wine and vinegar, it is better to avoid fruit pressing and perform the process with fruit mash. Inoculation is recommended for persimmon and is necessary for strawberry.
\end{abstract}

Keywords: wine, vinegar, fruit seasonings, acetic acid bacteria

\section{Introduction}

Every year, large amounts of fruit are wasted because the surplus cannot be consumed directly by the market and because some fruit does not fulfill market requirements (second- or third-quality fruit). Although some alternatives to direct consumption have already been implemented (jams, fruit concentrates, fruit juices, nectars, purées, etc) a large amount of fruit is still left in the fields to rot or be collected and later disposed of as waste. ${ }^{1}$ These practices create both an ecologic and an economic problem, because large amounts of organic matter have to be recycled and money must be spent on agrochemicals, labor force, and machinery for both the fruit that is consumed and the fruit that is disposed of, but all the costs are borne by the fruit that is consumed. Thus, higher prices and environmental contamination result from fruit surplus.

Other alternatives have been proposed and implemented in some countries, mostly transformations by fermentation. The resulting product, fruit wine, has a variable alcohol concentration and is often distilled because the market for fruit wines is not large. Some of the wines reported are, for instance, mango, ${ }^{2}$ banana, ${ }^{3}$ acerola, ${ }^{4}$ apricot, ${ }^{5}$ apple, ${ }^{6}$ gairoba, ${ }^{7}$ and are popular in some places. However, in a global alcoholic beverage market dominated by grape wine and beer, the impact of such wines is very limited because consumers are reluctant to try them. Furthermore, new alcoholic beverages are not very well received by consumers, and sometimes even have legal problems with being approved because they can cause health concerns for both the general public and the food safety authorities. 
On the other hand, the preservation of fruit components and their lack of transformation make fermentation one of the more environmentally friendly processes. Furthermore, transformation by fermentation can add some value because some of the microorganisms produce vitamins and other compounds that can improve the healthy components of fruit. Thus, the option of using fermented fruit is a good one if an alcohol content can be avoided. The transformation of ethanol into acetic acid fulfils some of these requirements because it maintains most of the fruit components, produces a stable product because of the acetic acid content and $\mathrm{pH}$ reduction, and can be used to season food directly or while cooking. Our alternative, then, is to use some fruits to produce food seasonings by double fermentation, alcoholic and acetous (or more appropriately acetic oxidation or acetification). Most of the knowledge available about these transformations has been generated by the wine and wine vinegar sector because it is a well known product and transformation process. ${ }^{8-10}$ The first transformation, alcoholic fermentation, is done by yeasts, especially Saccharomyces, although some nonSaccharomyces yeasts may also participate actively, at least in the early stages. ${ }^{11,12}$ Once the sugar has been converted into ethanol, the second process consists of an oxidation that is highly dependent on the availability of oxygen, which is needed by acetic acid bacteria. The amount of oxygen available is considerably reduced during alcoholic fermentation so, once the sugar has been exhausted and the oxygen concentration increases by aeration due to racking, pumping over, etc, acetification generally proceeds. ${ }^{13}$ Although most of the vinegar is produced from wine or alcohol, some fruit vinegars are also available. ${ }^{9}$

The aim of this work was to study whether fruit vinegar can be produced by two different processes (alcoholic fermentation and acetification) from persimmon and strawberry and if so, how to optimize the procedure. The kinetics of the process were analyzed in both spontaneous alcoholic fermentations and fermentations inoculated with commercial wine yeast. After the alcoholic fermentations, acetifications were also studied, but they were allowed to proceed with no further intervention.

\section{Materials and methods}

The study was carried out in 2008 using two different types of fruit, persimmon (Diospyros kaki, Sharoni variety) and strawberry (Fragaria ananasa, Camarosa variety). The fruit was picked in Huelva, Spain, during the season for each fruit (November for persimmon and April for strawberry).

\section{Conditions for producing persimmon and strawberry vinegar}

The vinegar was produced in a two-step process, first an alcoholic fermentation and then an acetification, which were carried out using crushed pulps of persimmon and strawberry. The fruit was cleaned (by removing the green parts) and crushed using a Philips HR 2094 liquidizer. To the crushed pulp, we added $60 \mathrm{mg} / \mathrm{L}$ sulfite and $3 \mathrm{~g} / \mathrm{hL}$ of pectolitic enzymes $(1.5 \mathrm{~g} / \mathrm{hL}$ of Depectil Clarification and $1.5 \mathrm{~g} / \mathrm{hL}$ of Depectil Extra Garde FCE, Martin Vialatte Oenologie, France).

For each fruit, two different processes were carried out in triplicate experiments. One of the processes was a spontaneous alcoholic fermentation followed by spontaneous acetification (in both cases natural microbiota were allowed to proliferate). In the other process, the alcoholic fermentation was inoculated at the beginning with the commercial Saccharomyces cerevisiae wine strain QA23 (Lallemand, Inc., Canada) at a concentration of $2 \times 10^{6}$ cells $/ \mathrm{mL}$. In this case, acetification also proceeded without acetic acid bacteria inoculation.

In the case of strawberry, the process was repeated in single experiments (spontaneous and inoculated) using the liquid obtained after crushing and pressing the fruit pulp. The strawberries were crushed in the same way as above, but were pressed in a $10 \mathrm{~L}$ vertical press. The experiments were as above, with the only difference being that pressed juice was used instead of mashed pulp.

The 14 processes (six for persimmon and eight for strawberry) were conducted under laboratory conditions in $8 \mathrm{~L}$ glass containers with a broad top hole of $10 \mathrm{~cm}$ in diameter. During alcoholic fermentation and acetification, this top hole was covered by a cloth to keep out insects, dust, etc. The glass containers were filled with $6 \mathrm{~L}$ of the initial pulp or liquid, leaving an air chamber of $2 \mathrm{~L}$. To fill the containers in triplicate, a total of $50 \mathrm{~kg}$ of persimmon and $65 \mathrm{~kg}$ of strawberry was required. When strawberry was pressed, we required $9 \mathrm{~L}$ of crushed strawberry pulp to obtain $6 \mathrm{~L}$ of liquid. As a general criterion, alcoholic fermentation was considered to have finished when the sugar had been consumed $(<2 \mathrm{~g} / \mathrm{L})$ and acetification finished when the ethanol concentration had fallen below $1 \%(\mathrm{v} / \mathrm{v})$. All fermentations were done at room temperature $\left(23 \pm 3^{\circ} \mathrm{C}\right)$.

\section{Chemical analysis}

The vinegar production processes were also studied at room temperature. Temperature, $\mathrm{pH}$, and concentration of free 
amino nitrogen (FAN), sugars, ethanol, and acetic acid were analyzed throughout the processes. Temperature was measured using a digital thermometer (Hanna, HI 145-00) and pH using a pH meter (Crison, micro-pH 2002). FAN concentration was analyzed using the formol index method. ${ }^{14}$ Sugar concentrations (glucose, fructose, and sucrose) and ethanol were measured with enzymatic kits (Boehringer Mannheim, Germany). Acidity was determined by titration with $0.5 \mathrm{~N}$ $\mathrm{NaOH}$ and phenolphthalein as the indicator. ${ }^{15}$

\section{Microbial analysis}

Imposition of the inoculated yeast was analyzed during the alcoholic fermentations. Samples of the spontaneous and inoculated processes were taken at the beginning, middle, and end of the fermentation and plated on a YEPD solid medium made of glucose $20 \mathrm{~g} / \mathrm{L}$, peptone $20 \mathrm{~g} / \mathrm{L}$, yeast extract $10 \mathrm{~g} / \mathrm{L}$, and agar $20 \mathrm{~g} / \mathrm{L}$ (Cultimed, Barcelona, Spain). Twenty colonies of each point were analyzed by pattern comparison of mtDNA restriction fragment length polymorphisms. ${ }^{16}$

\section{Statistical analysis}

Each fermentation condition was performed in triplicate. The data were statistically analyzed using the SPSS 17 software package. By Student $t$-test, we determined the differences between the inoculated and spontaneous fermentations (the statistical level of significance was set at $P \leq 0.05$ ).

\section{Results}

We studied the production of vinegar from two fruits, ie, persimmon and strawberry. All the processes were carried out with crushed fruit. The fresh pulp was also pressed in some of the experiments with strawberry. In order to analyze the vinegar process and to prevent side effects caused by wood, we used glass containers cleaned with boiling water and bleach.

\section{Alcoholic fermentation}

The initial sugar concentration of the persimmon fruit mash was $110.1 \mathrm{~g} / \mathrm{L}$. The amount of sucrose was rather low in comparison with that of fructose and glucose (see Table 1). The initial $\mathrm{pH}$ was 5.5 and decreased sharply to 3.8 after 24 hours in both the spontaneous and inoculated alcoholic fermentations. This value remained constant throughout the alcoholic fermentation. FAN was not a limiting factor for alcoholic fermentation, yet in all cases it was completely consumed. Inoculated alcoholic fermentation was faster than the spontaneous process (Figure 1a) because the lag phase
Table I Chemical analysis of the persimmon and strawberry pulp before vinegar production

\begin{tabular}{lll}
\hline Parameter & Persimmon & Strawberry \\
\hline Total sugars & 110.1 & 28.4 \\
Fructose & 44.8 & 15.8 \\
Glucose & 57.3 & 8.3 \\
Sucrose & 8 & 4.3 \\
FAN $(\mathrm{mg} / \mathrm{L})$ & 120 & 224 \\
pH & 5.5 & 3.5 \\
Titratable acidity $(\%, \mathrm{w} / \mathrm{v})$ & 0.6 & 0.9 \\
Ethanol $(\%, \mathrm{v} / \mathrm{v})$ & - & 1.4 \\
\hline
\end{tabular}

Abbreviation: FAN, free amino nitrogen.

was shorter and the fermentation rate similar. Furthermore, the alcohol concentration was $0.5 \%(\mathrm{v} / \mathrm{v})$ higher in the inoculated fermentation.

The strawberry fruit mash contained a very low initial sugar concentration ( $28.4 \mathrm{~g} / \mathrm{L}$, see Table 1$)$, which was mostly fructose. In order to proceed with alcoholic fermentation, we added sucrose to a final sugar concentration of $100 \mathrm{~g} / \mathrm{L}$. The $\mathrm{pH}$ was 3.5 and remained constant throughout the alcoholic fermentation. FAN was high, yet it was also completely consumed. Overripe fruits were transported to the laboratory in good and healthy condition, although some alcohol had already been produced, probably due to some alcoholic maceration. The alcohol content of the fruit mash was $1.4 \%$ $(\mathrm{v} / \mathrm{v})$. Again, the inoculated fermentation was faster than the spontaneous process, in which the lag phase was very long and the fermentation rate similar (Figure 1b). The same occurred when the strawberry juice was fermented, rather than the crushed pulp, in an identical process (Figure 1c). The levels of alcohol were similar in this case.

In all cases, the inoculated strain took over the alcoholic fermentation process with a presence over $80 \%$ in the recovered colonies for all the sampling points in the inoculated fermentations, whereas it was always absent in the spontaneous ones. The starter was the only yeast recovered at the end of the inoculated fermentations. The yeast populations achieved levels of $2 \times 10^{7}$ colony-forming units $/ \mathrm{mL}$ in all cases, with a longer lag phase (two days) in the spontaneous fermentations than in the inoculated ones (less than 24 hours).

\section{Acetification}

Independently of the alcoholic fermentation, the acetification process in the persimmon fruit proceeded similarly (Figure 2a). The overall acetification process finished in 30 days and in both cases, the acetic acid content was $4.5 \%$ $(\mathrm{w} / \mathrm{v})$. In both processes, the $\mathrm{pH}$ decreased to 3.4. 


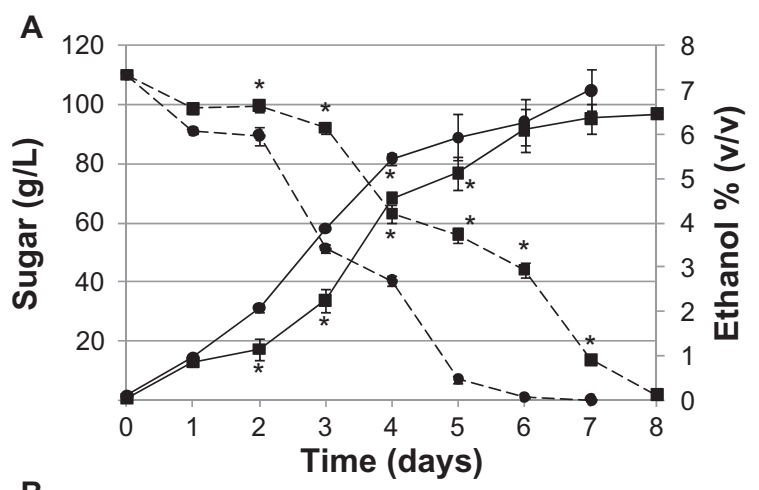

B

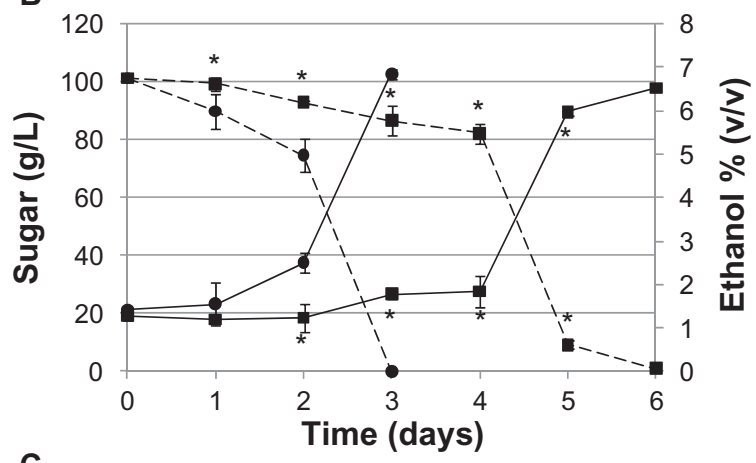

。

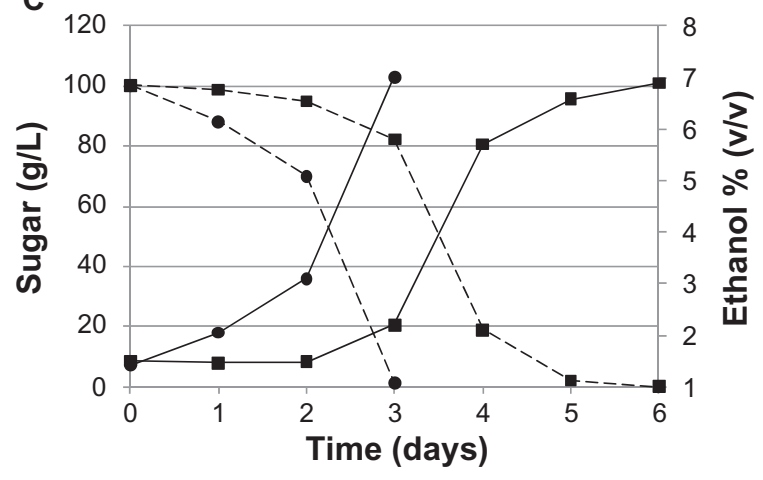

Figure I Development of alcoholic fermentation (a, persimmon; b, strawberry; c, pressed strawberry). Sugar consumption (---) and ethanol production (-) during spontaneous $(\boldsymbol{(})$ and inoculated $(\bullet)$ alcoholic fermentations.

Note: *Statistically significant differences $P \leq 0.05$

The initial titratable acidity at the end of alcoholic fermentation of the strawberry was $0.9 \%(\mathrm{w} / \mathrm{v})$ in all cases. However, only four of the six glass vessels of crushed pulp successfully completed the acetification (two in each experiment). The other two containers also consumed the ethanol, yet no increase in the titratable acidity was observed ( $1 \%(\mathrm{w} / \mathrm{v})$ final titratable acidity, data not shown). The successful strawberry acetifications took longer than for persimmon when crushed fruit was used (70 days for strawberry versus 30 days for persimmon, Figures $2 \mathrm{a}$ and $2 b)$. For strawberry, the yeast inoculation had a similar lack of effect because all the acetifications proceeded in parallel and similar amounts of acetic acid were recovered. The $\mathrm{pH}$
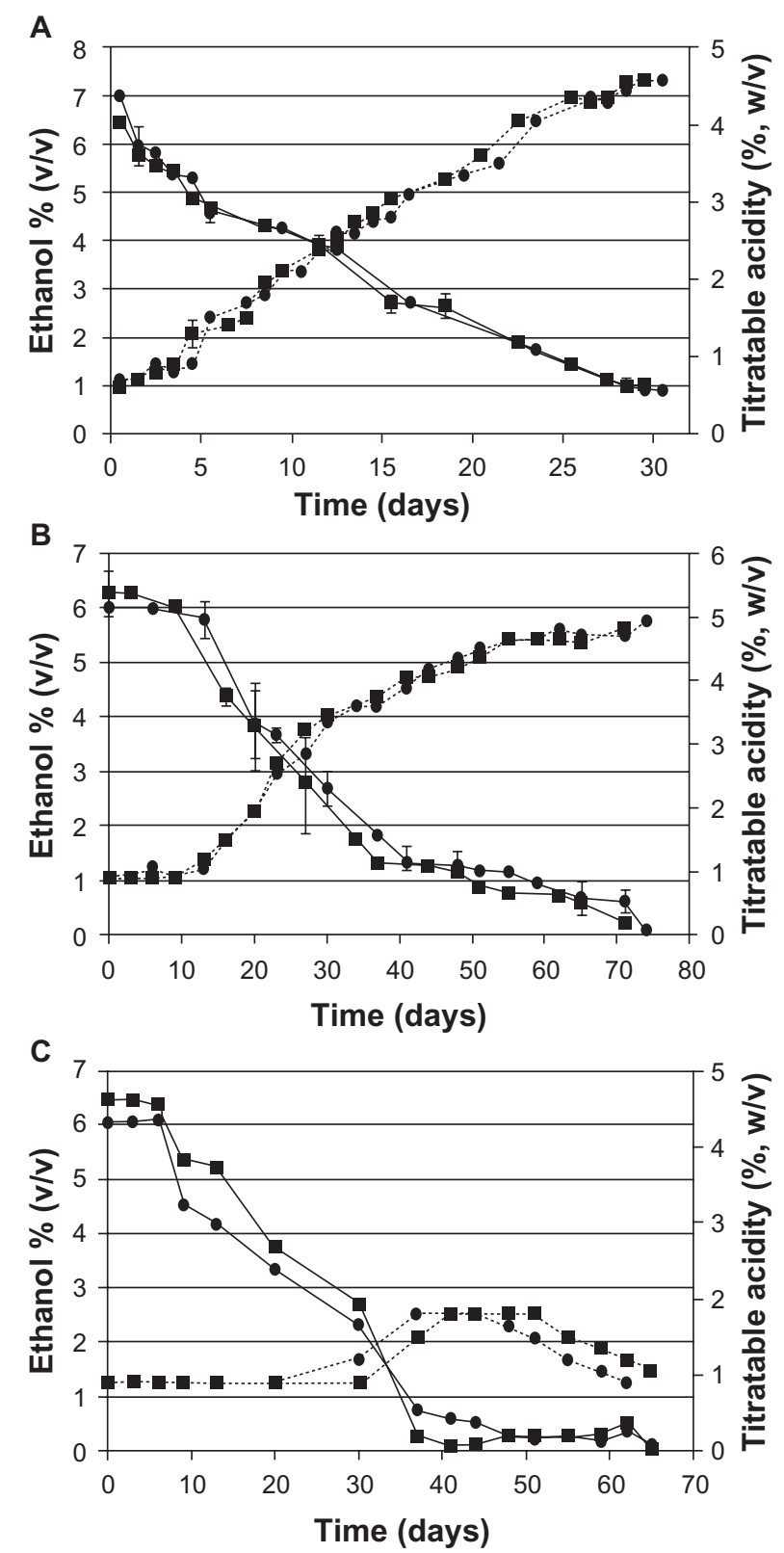

Figure 2 Development of acetification (a, persimmon; b, strawberry; c, pressed strawberry). Ethanol consumption (---) and titratable acidity production $(-)$ during spontaneous ( $\boldsymbol{(})$ and inoculated $(\bullet)$ acetifications.

values decreased to 3.1 only in the successful acetifications, while in the others, the $\mathrm{pH}$ was the same as that observed at the end of alcoholic fermentation (3.5). The acetification performed with the pressed strawberries also showed poor acetic acid production (Figure $2 \mathrm{c}$ ). The titratable acidity was only $1.8 \%(\mathrm{w} / \mathrm{v})$ after 41 days, and the acetic acid concentration remained constant for 10 days before finally decreasing to $1 \%(\mathrm{w} / \mathrm{v})$ after 14 more days. However, the ethanol concentration decreased to below $1 \%(\mathrm{v} / \mathrm{v})$ after 65 days. The $\mathrm{pH}$ value decreased slightly to 3.4 . 


\section{Fruit vinegar yield}

Fruit vinegar was only obtained when fruit pulp was used. However, the pulp was still dense and had to be pressed to remove solid debris and obtain a clear product. Despite the obvious differences between the two processes, the vinegar yields were similar. In terms of percentage of final product (liquid vinegar, in L) obtained per initial amount of fruit (in $\mathrm{kg}$ ) the results obtained with persimmon were $64.8 \pm 5.4 \%$ for the inoculated process and $63.2 \pm 2.9 \%$ for the spontaneous process. For the strawberry vinegar, the yield was only calculated for those processes in which the acetic acid was about $5 \%(\mathrm{w} / \mathrm{v})$, and the value was $66.2 \pm 2.5 \%$. In terms of liquid recovery after pressing, these values are similar to those observed when the fresh strawberry was pressed and strawberry juice was obtained (66.6\%).

\section{Discussion}

Our knowledge of the alcoholic fermentation of fruit is influenced a great deal by the fermentations of the wine and beer industry, in which the limiting parameters are mostly lack of equilibrium between fermentable sugar and available nitrogen and the availability of different vitamins or minerals. ${ }^{13}$ Furthermore, the use of selected yeast is a requirement and makes a significant contribution to the characteristics of the final product for beer. In wine, however, it is not so necessary, but it is a very common practice, especially after the development of active dry wine yeast technology. ${ }^{17}$ Even so, spontaneous fermentations performed with natural wild yeasts present on the surface of grapes or the winery equipment still have their advocates because of the authenticity of the final products. ${ }^{18}$ For both beer and wine, most of the yeasts available for starting cultures have been selected from brewing or winemaking because they are good performers, have low nutritional requirements, start fermentations quickly, provide good fermentation rates, and produce byproducts that are appreciated by consumers. ${ }^{17}$ Furthermore, because a particular yeast can give the final product uniform characteristics, it is also common practice to select a local wine yeast. ${ }^{12,18}$ However, no yeast is available for the fermentation of other fruit, so most of the fermentation is performed with wine yeast or spontaneously. The present study has made a detailed analysis of both processes (inoculated and spontaneous fruit fermentation) in order to obtain vinegars instead of wines after acetification of the initial wines. We had no problems with alcoholic fermentation, whether spontaneous or inoculated. The fruits used (strawberry and persimmon) have a more balanced amount of available nitrogen, considering the amount of fermentable sugar compared with that in grapes. Other nutrients and vitamins are also available in both fruits. ${ }^{19,20}$ In both cases, the inoculated fermentation proceeded faster than the spontaneous process, as happens with wine, ${ }^{21}$ largely because of the shorter lag phase when the active yeast is seeded. In regular transformation into wine, sugar is expected to convert into ethanol (approximately $17 \mathrm{~g}$ sugar produces $1 \%$ ethanol $\mathrm{v} / \mathrm{v}) \cdot{ }^{13}$ Interestingly, no differences were observed in strawberry fermentation with pressed fruit and fruit mash. It should be pointed out that overripe strawberries produced a certain amount of alcohol, as observed in the alcoholic maceration of grapes, where the alcohol content in the process easily produces over $2 \%$ ethanol $(\mathrm{v} / \mathrm{v}) .^{13,22}$

Although yeast inoculation was not actually needed to produce these fruit wines, specific yeast for inoculation would be highly recommended in the industrialization of both the wine and vinegar processes. Industrialization requires shorter production periods and a repetitive production process, which could be obtained by the practice of inoculating selected strains.

While alcoholic fermentation of grapes is a well-known process, the acetification process is still only partially understood. Most of the vinegar is produced from alcohol and a mix of nutrients in industrial processes whereby the seed culture is submerged in a highly aerated vat and maintained continuously throughout a batch process, with a daily refilling system. Wine vinegar can also be produced in this way, although high-quality vinegars are produced with the traditional surface culture method. Using this method, the acetic acid bacteria lie on the liquid-air surface and produce a biofilm that uses oxygen directly from the air or from the limited amounts of air that pass through the wood pores. However, most starter cultures in both cases have very limited availability and are poorly characterized. ${ }^{23}$ In fact, most industrially available cultures come in liquid form as mixed, noncharacterized cultures and require some time to perform acetification. In contrast, traditional methods use the "vinegar mother", as a starter culture. This vinegar mother consists of vinegar in progress or the biofilm that is on the surface of vinegar. However, it is well known that acetic acid bacteria are part of the natural microbiota of grapes and wines, and they often survive until the end of alcoholic fermentation. ${ }^{24}$ In our case, we allowed the natural population of acetic acid bacteria to acetify the fruit wines. We had no problem with the persimmon fruit, and the acetification proceeded at a reasonable rate with the natural microbiota present in the fruit, as already observed by other authors. ${ }^{25}$ Wine acetification with traditional methods is much slower, probably due to a higher 
alcohol concentration (10-15\% ethanol v/v) in the starting wine. ${ }^{26}$ In fact, most of the wine used for vinegar production is diluted with water or vinegar. In strawberry, although the starting alcohol concentration was similar, the acetification was much slower and in some cases, did not finish or produce acetic acid. In fact, if we bear in mind that the pressed strawberry is less acetified than the fruit mash, it is easy to draw the conclusion that some nutrients in the solid particles of the mash are needed for the acetic acid bacteria to perform well. Therefore, it is evident that to produce strawberry vinegar, the wine composition needs to be analyzed and appropriate starter cultures need to be used because there is a high risk of unfinished acetifications.

Our yield in terms of the final product (wine or vinegar) was acceptable because it was always well over $60 \%$. We performed the whole process at the laboratory level, with such limiting factors as the strength of the press and the recovery of fruit pulp on a small scale. Scaling up to higher volumes and with industrial equipment will produce higher yields, similar to those observed for wine. The final product obtained in both cases showed good color (pink for strawberry, pale yellow for persimmon) and good organoleptic characteristics. The strawberry vinegar had a strong strawberry flavor, which compensated for the pungent smell from the volatile acidity, and proved to be a very promising product. Further chemical characterization of both products is under way.

From the technologic point of view, most of the protocols used in wine and wine vinegar production can be used to produce persimmon and strawberry wine and vinegar. However, initial pressing of the fruit is not recommended because of the firmness of the fruit (persimmon) and the lack of some characteristics required for vinegar production (strawberry). Although the alcoholic fermentation and acetification of persimmon proceeded at good rates and took an acceptable length of time, the use of selected starter cultures is recommended to shorten this time and increase the safety of the product. In strawberry, although starter cultures are not essential for alcoholic fermentation, they are required for producing vinegar repetitively and efficiently. In our laboratory, we are now analyzing the possible use of native microbiota associated with persimmon and strawberry as starter cultures for both alcoholic fermentation and acetification.

\section{Acknowledgments}

The present work was part of the AGL2007-66417-C02-02/ ALI project financed by the Spanish Ministry of Education and Science. The authors thank the Language Service of the Rovira i Virgili University for revising the manuscript.

\section{Disclosure}

The authors report no conflict of interest in this work.

\section{References}

1. Grewal HS, Tewari HK, Kalra KL. Vinegar production from substandard fruits. Biol Waste. 1988;26:9-14.

2. Reddy LVA, Reddy OVS. Production and characterization of wine from mango fruit (Mangifera indica L.). World J Microbiol Biotechnol. 2005;21:1345-1350.

3. Akubor PI, Obio SO, Nwadomere KA, Obiomah E. Production and quality evaluation of banana wine. Plant Foods Hum Nutr. 2003;58:1-6.

4. Santos SC, Almeida SS, Toledo AL, Santana JCC, de Souza RR. Making and sensorial analysis of Malpighia Punicifolia L. wine. Braz J Food Technol. 2005;10:47-50.

5. Joshi VK, Bhutani VP, Sharma RC. Effect of dilution and addition of nitrogen source in chemical, mineral and sensory qualities of wild apricot wine. Am J Enol Vitic.1990;41:229-231.

6. Joshi VK, Sandhu DK, Attri BL, Walla RK. Cider preparation from apple juice concentrate and its consumer acceptability. Indian J Hort. 1991;48:321.

7. Duarte WF, Dias DR, de Melo Pereira GV, Gervásio IM, Schwan RF. Indigenous and inoculated yeast fermentation of gabiroba (Campomanesia pubescens) pulp for fruit wine production. J Ind Microbiol Biotechnol. 2009;36:557-569

8. Adams MR, Moss MO. Food Microbiology. 2nd ed. Cambridge: Royal Society of Chemistry; 2000.

9. Plessi M. Vinegar. In: Caballero B, Trugo LC, Finglas PM, editors. Encyclopedia of Food Sciences and Nutrition, 2nd ed. Oxford: Academic Press; 2003.

10. Raspor P, Goranovic D. Biotechnological applications of acetic acid bacteria. Crit Rev Biotechnol. 2008;28:101-124.

11. Constantí M, Poblet M, Arola Ll, Mas A, Guillamón JM. Analysis of yeast populations during alcoholic fermentation in a newly established winery. Am J Enol Vitic. 1997;48:339-344.

12. Torija MJ, Rozès N, Poblet M, Guillamón JM, Mas A. Yeast population dynamics in spontaneous fermentations: Comparison between two different wine producing areas over a period of three years. Antonie van Leeuwenhoek. 2001;79:345-352.

13. Ribéreau-Gayon P, Dubourdieu D, Donèche B, Lonvaud A. Handbook of Enology. The Microbiology of Wine and Vinifications. West Sussex: Wiley; 2006.

14. Aerny J. Nitrogen compounds of musts and wines. Rev Suisse Vitic Arbor Hortic. 1996;28:161-165.

15. Ough CS, Amerine MA. Methods for Analysis of Musts and Wines. California: Wiley-Interscience; 1987.

16. Querol A, Barrio E, Huerta T, Ramón, D. 1992. Molecular monitoring of wine fermentations conducted by dry yeast strains. Appl Environ Microbiol. 1992;58:2948-2952.

17. Degre R. Selection and commercial cultivation of wine yeast and bacteria. In: Fleet G, editor. Wine Microbiology and Biotechnology. London: Taylor and Francis; 1993.

18. Fleet $\mathrm{G}$. The microorganisms of winemaking-isolation, enumeration and identification. In: Fleet G, editor. Wine Microbiology and Biotechnology. London: Taylor and Francis; 1993.

19. Celik A, Ercisli S. Persimmon cv. Hachiya (Diospyros kaki Thunb.) fruit: Some physical, chemical and nutritional properties. Int $J$ Food Sci Nutr. 2008;59:599-606.

20. Kallio H, Hakala M, Pelkkikangas AM, Lapveteläinen A. Sugars and acids of strawberry varieties. Eur Food Res Technol. 2000;212: $81-85$.

21. Constantí M, Reguant C, Poblet M, Zamora F, Mas A, Guillamon JM. Molecular analysis of yeast population dynamics: Effect of sulphur dioxide and the inoculum in must fermentation. Int J Food Microbiol. 1998;41:169-175. 
22. Divies C. Bioreactor technology and wine fermentation. In: Fleet G, editor. Wine Microbiology and Biotechnology. London: Taylor and Francis; 1993.

23. Mas A, Torija MJ, González A, Poblet M, Guillamón JM. Acetic acid bacteria in oenology. Contributions to Science. 2007;3:511-521.

24. González A, Hierro N, Poblet M, Mas A, Guillamón JM. Application of molecular methods to demonstrate species and strain evolution of acetic acid bacteria population during wine production. Int J Food Microbiol. 2005;102:295-304.
25. Lee SH, Kim JC. A comparative analysis for main components change during natural fermentation of persimmon vinegar. J Korean Soc Food Sci Nutr. 2009;38:372-376.

26. Torija MJ, Mateo E, Vegas CA, et al. Effect of wood type and thickness on acetification kinetics in traditional vinegar production. Int $J$ Wine Res. 2009;1:155-160.
International Journal of Wine Research

\section{Publish your work in this journal}

The International Journal of Wine Research is an international, peer-reviewed open-access, online journal focusing on all scientific aspects of wine, including: vine growing; wine elaboration; human interaction with wine; and health aspects of wine. The journal provides an open access platform for the reporting

\section{Dovepress}

of evidence based studies on these topics. The manuscript management system is completely online and includes a very quick and fair peer-review system, which is all easy to use. Visit http://www.dovepress.com/testimonials.php to read real quotes from some of our published authors.

Submit your manuscript here: http://www.dovepress.com/international-journal-of-wine-research-journalisease-journal 